\title{
STC-M0X-Th: Um Novo Código Termo-Hidráulico para Pesquisa e Ensino
}

\author{
Thiago Augusto dos Santos'; Giovanni Laranjo de Stefanil
}

凹thia.santos@hotmail.com

1. Instituto de Pesquisas Energéticas e Nucleares, IPEN/USP. São Paulo - Brasil.

\section{Histórico do Artigo:}

Recebido em: 22 de novembro de 2019 Aceito em: 08 de julho de 2020 Publicado em: 31 de agosto de 2020

Resumo: 0 trabalho trata da criação de um programa elaborado em ambiente MATLAB que calcula os limites térmicos de projeto de um típico reator a água pressurizada (PWR), que é a temperatura central da pastilha combustivel e a taxa de ebulição nucleada (DNBR). Outras distribuições de temperatura e grandezas hidrodinâmicas do líquido refrigerante, como a entalpia e a queda de pressão também são calculadas. 0 código possui peculiaridades, como o fato de permitir cálculos com combustíveis de $\mathrm{UO}_{2}$ puro e proporções do óxido misto de Urânio/Tório - MOX (U,Th). Estas, além da sua interface amigável com o usuário provam que o código pode ser utilizado em trabalhos de pesquisa , bem como em disciplinas de graduação e pós graduação voltadas ao estudo de termo-hidráulica de reatores nucleares em cursos de graduação e pós graduação de engenharia (nuclear e/ou da energia) espalhados pelos país, como no caso do curso de graduação de Engenharia de Energia da Universidade Federal do ABC, onde é uma disciplina optativa. Para a validação do código foram utilizados dados do reator AP1000 da Westinghouse. 0 programa se apresentou com comportamento físico dentro do esperado para o modelo, gerando resultados confiáveis para eventuais projetos de reatores (validado com dados experimentais e outros programas), bem como propicia a alunos uma experiência diferenciada dentro da aprendizagem dos conceitos empregados na área, uma vez que o programa permite uma análise mais profunda de determinados conceitos na área de termo-hidráulica que dentro da aula expositiva e com exercícios convencionais não poderiam ser explorados.

Palavras-chave: Ensino de Engenharia, Reatores Nucleares, MATLAB, Termo-hidráulica.

\section{STC-M0X-Th: A New Thermal Hydraulic Code for Research and Education}

Abstract: STC-MOX-Th (Simplified Thermal - Hydraulics Code-Mixed 0xide Thorium) is a program developed in a MATLAB environment that calculates the thermal design limits of a typical Pressurized Water Reactor (PWR), which is the temperature fuel pellet center and the Nuclear Boiling Ratio (DNBR). In addition, other temperature distributions and hydrodynamic coolant quantities such as enthalpy and pressure drop are also calculated. The present work presents the code and shows its peculiarities, such as the fact that it allows calculations with fuels of pure $\mathrm{U}_{2}$ and proportions of mixed oxide of Uranium / Thorium - MOX (U, Th). These, besides its user-friendly interface, among other characteristics to be presented later, prove that the code can be used in research projects, as well as in disciplines focused on the study of thermohydraulics of nuclear reactors in engineering courses, as in the case of the undergraduate course in Energy Engineering at the Federal University of ABC, where it is an elective course. For data validation, data from Westinghouse's AP-1000 reactor were used. The program showed physical behavior as expected for the model, generating reliable results for any reactor projects (validated with experimental data and other codes), as well as providing students with a more complete experience in the learning of the concepts employed in the area.

Keywords: Engineering Teaching, Nuclear Reactors, MATLAB, Thermal-hydraulics. 


\section{STC-M0X-Th: Un Nuevo Código Hidráulico Térmico para la Investigación y la Educación}

Resumen: STC-MOX-Th (Simplified Thermal - Hydraulics Code-Mixed 0xide Thorium) es un programa desarrollado en un entorno MATLAB que calcula los límites de diseño térmico de un Reactor de agua a presión (PWR) típico, que es el centro de pellets de combustible de temperatura y el Nuclear Ratio de ebullición (DNBR). Además, también se calculan otras distribuciones de temperatura y cantidades de refrigerante hidrodinámico, como la entalpía y la caída de presión. El presente trabajo presenta el código y muestra sus peculiaridades, como el hecho de que permite realizar cálculos con combustibles de U02 puro y proporciones de óxido mixto de uranio/torio - MOX (U, Th). Estos, además de su interfaz fácil de usar, entre otras características que se presentarán más adelante, demuestran que el código se puede utilizar en proyectos de investigación, así como en disciplinas centradas en el estudio de la termohidráulica de reactores nucleares en cursos de ingeniería, como en el caso del curso de pregrado en Ingeniería Energética en la Universidad Federal de ABC, donde es un curso electivo. Para la validación de datos, se utilizaron datos del reactor AP-1000 de Westinghouse. El programa mostró un comportamiento físico como se esperaba para el modelo, generando resultados confiables para cualquier proyecto de reactor (validado con datos experimentales y otros códigos), así como brindando a los estudiantes una experiencia más completa en el aprendizaje de los conceptos empleados en el área.

Palabras clave: Enseñanza de Ingeniería, Reactores Nucleares, MATLAB, Termohidráulica.

\section{INTRODUÇ̃̃o}

0 estudo de termo-hidráulica de reatores é parte integrante de qualquer curso de engenharia nuclear. A área tem notável importância, sobretudo no que diz respeito à análise de acidentes em reatores nucleares. Possui um elevado nível de complexidade, sendo esta uma aplicação conjunta de diversas áreas da engenharia como a mecânica dos fluidos, transferência de calor e massa, termodinâmica, entre outras. 0 que exige certo grau de maturidade de um aluno de engenharia.

Segundo Masterson (2017) um núcleo de um reator de água pressurizada (PWR) usa água leve (água comum destas de torneira, altamente destilada) tanto para o moderador quanto para o líquido de arrefecimento, e é mantido sob pressão considerável (cerca de 15,5 MPa ou 2250 PSI) para impedir que ferva. Ele entra no núcleo a cerca de $290^{\circ} \mathrm{C}$ e deixa o núcleo a cerca de $325^{\circ} \mathrm{C}$. Deve-se aos geradores de vapor, onde parte do calor é removida e, em seguida, é bombeada de volta para o núcleo a partir de baixo. A passagem da água sobre as varetas de combustível aumenta sua temperatura média em cerca de $35^{\circ} \mathrm{C}$. Sua densidade também diminui à medida que é aquecida, e pode haver entre dois e quatro geradores de vapor conectados ao circuito primário.

Segundo Todreas e Kazimi (1990) no projeto de um reator PWR, os cálculos neutrônicos, que fornecem a densidade de potência presente no reator, devem ser checados de modo a não 
violarem os limites térmicos do reator projetado. Dentre esses limites estão, por exemplo, o ponto de fusão do material combustível, que uma vez atingido causa um acidente de proporções catastróficas.

Para efetuar tais cálculos, inúmeros códigos já foram desenvolvidos. COBRA, Rowe (1970) e THINC, Zernick et al. (1962) são exemplos de códigos da área de termo-hidráulica consolidados e muito utilizados em diversos trabalhos no mundo acadêmico. Tais códigos são extremamente complexos e permitem uma análise termo hidráulica de um reator nuclear. Porém, códigos como esses são extremamente caros, nem sempre realizam cálculos em certas condições específicas e podem não ter uma interface amigável com o usuário. Por esses motivos, softwares de análise termo-hidráulica mais simples são desenvolvidos em diversas universidades. 0 trabalho de Junior (2010) é um exemplo dessa prática.

Para Belhot et al. (2001), a inserção de códigos computacionais como instrumentos auxiliares na aprendizagem vem sendo cada vez mais recomendada e utilizada no Brasil, país que ainda tem a educação em engenharia apoiada na acumulação de conteúdo e na reprodução do conhecimento.

Dentro dos dois propósitos (pesquisa e ensino) foi criado por Santos (2016) na Universidade Federal do ABC (UFABC) um código computacional, simplificado, intitulado de STCMOX-Th (Simplified Thermal- hydraulicsCode-Mixed 0xide Thorium), que avalia os limites térmicos de um reator nuclear a água pressurizada (Pressure Water Reactor-PWR) que utiliza pastilhas combustíveis de U02 ou combustíveis de óxidos mistos, que são combustíveis que combinam Urânio com outro actinídeo, como o Plutônio ou o Tório. Além disso, o código fornece parâmetros hidráulicos, como a queda de pressão sobre o líquido refrigerante (neste caso, água) ao longo da vareta combustível.

0 código atuou como ferramenta para os cálculos termo-hidráulicos do projeto de um reator com um combustível de óxidos mistos de Urânio-Tório, APTh-1000, mostrado em Maiorino et al. (2017). Além disso, também foi utilizado nas aulas da disciplina de termo-hidráulica de reatores nucleares, que faz parte da grade curricular no curso de graduação em Energia da UFABC, sendo esta uma disciplina optativa. Esse trabalho tem como objetivo mostrar a versatilidade do código STC-MOX-Th, podendo ser utilizado em atividades tanto relacionadas à pesquisa, quanto ao ensino.

Revista Internacional de Ciências, v. 10, n. 02, p. 3-22, mai-ago, 2020 


\section{MATERIAIS E MÉTODOS}

\section{Descrição do código}

0 STC-MOX-Th é um código desenvolvido em linguagem MATLAB que analisa o comportamento térmico utilizando dados coletados do canal mais quente do núcleo de um reator nuclear PWR, como os reatores ANGRA I e II, por exemplo. Nesse tipo de reator, o combustível tem forma de uma pastilha e está armazenado em varetas no núcleo do reator, conforme disposição mostrada na figura 1, a fim de realizar as reações de fissão que gerarão energia.

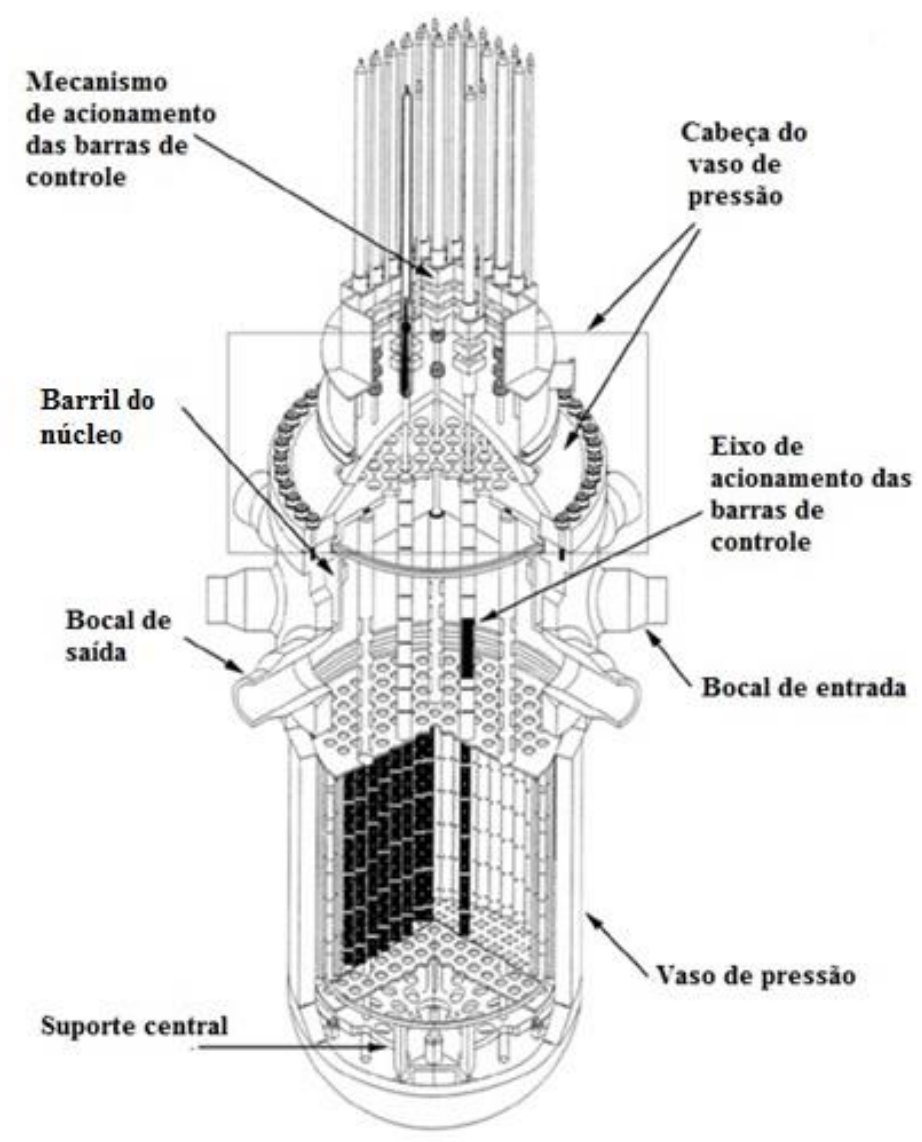

Figura 1. Vaso de pressão de um reator PWR detalhando o núcleo.

Fonte: USNRC (2017).

Apesar da relativa simplicidade em comparação a códigos como o COBRA, o STC-MOX-Th respeita as leis de conservação de massa e energia e os cálculos são realizados no canal mais quente. Possui uma parte hidráulica extremamente simples, porém obedecendo as leis de conservação de massa, momento e energia. Sua confiabilidade foi testada por Cunha et al. (2019) comparando os resultados com códigos já bem considados na área e com dados da 
Westinghouse por Santos et al. (2017) (2016) e mais detalhadamente na dissertação de mestrado de Santos (2016). A parte térmica é voltada a resolução da equação da condução térmica nas regiões da pastilha combustível e do revestimento e para regiões compreendidas por fluidos, a Lei de resfriamento de Newton é utilizada.

0 STC-MOX-Th tem como vantagens a interface amigável ao usuário, mostrada na figura 2, e, apesar da simplicidade, é capaz de fornecer resultados com confiabilidade. Para isso foram utilizados dados de reatores de geração 2 de companhias como Westinghouse, Babcock \& Wilcox e Combustion Engineering a fim de validar o código.

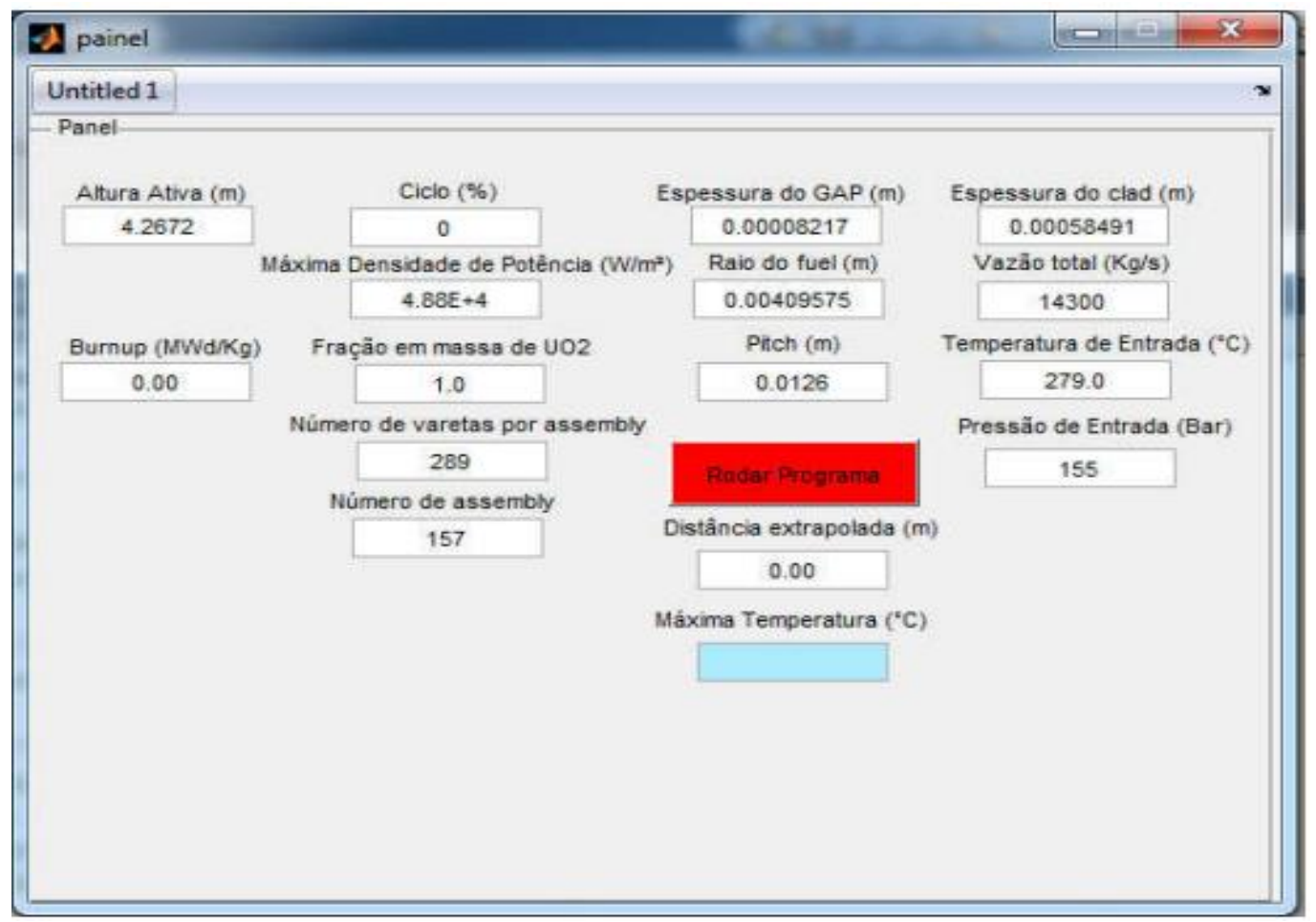

Figura 2. Interface STC-MOX-Th Fonte: Santos (2016).

Soma-se às vantagens citadas anteriormente o fato do código ser criado numa universidade, e assim proporcionar um ganho no ponto de vista financeiro para a mesma. É comum que códigos voltados para cálculos como os mostrados neste trabalho possuam licenças demasiadamente caras, apesar da plataforma MatLab não ser gratuita, uma versão opensource similar chamada 0ctave é disponível gratuitamente para linux e compatível com os scripts de MatLab. Do ponto de vista educacional, o código auxilia na aprendizagem de conteúdos extremamente complexos, que envolvem diversas áreas do ramo das engenharias. Para isso, o código fornece em sua saída de dados, um arquivo em Excel que contém grandezas como a capacidade térmica, a entalpia e a densidade da água ao longo da vareta. Tais grandezas não 
possuem tanta relevância no projeto do núcleo de um reator nuclear, mas tornam-se úteis quando analisadas num contexto mais voltado ao ensino de engenharia.

Outro ponto que mostra a versatilidade do código são os tipos de combustíveis que podem ser utilizados e o regime de queima (que será discutido mais adiante) que os mesmos se encontram. Pouquíssimos códigos realizam cálculos termo-hidráulicos utilizando óxidos mistos, sendo que os que possuem esse recurso limitam-se ao combustível de Urânio-Plutônio. A utilização de combustíveis de Urânio-Tório é um ganho tanto na pesquisa, como em disciplinas de graduação e pós-graduação de engenharia nuclear.

\section{Análise Hidráulica}

0 objetivo da análise hidráulica é fornecer as distribuições de velocidade e pressão com base no tipo de escoamento em certa geometria sob condições iniciais e de contorno especificadas, garantindo a conservação de massa, momento e energia, com base em Todreas e Kazimi (1990). 0 ponto principal do código, no que diz respeito à parte hidráulica, é a de determinar a queda de pressão ao longo do canal analisado em seu eixo axial.

A análise hidráulica parte da segunda lei de Newton em relação a um sistema de coordenadas iniciais, e deste chega-se à equação diferencial da conservação do momento no eixo axial, no estado estacionário, voltado a um tubo vertical, como mostrado em Fox et al. (2006).

$-\frac{d p}{d z}=\frac{d}{d z}\left(\frac{G^{2}}{\rho}\right)+\frac{1}{A_{Z}}\left(\int_{P_{Z}} \tau_{w} d P_{Z}\right)+\rho g$.

(1)

Onde p é a pressão, $\mathrm{P}_{\mathrm{z}}$, o momento linear referente ao eixo vertical, G, o fluxo mássico, $\mathrm{A}_{\mathrm{z}}$, a área do canal, $\tau_{W}$, a tensão de cisalhamento nas paredes da vareta, $\rho$, a densidade do fluido, a aceleração da gravidade $g$ e $\theta$ é o ângulo de inclinação da vareta em relação ao eixo horizontal. Cada termo da equação (1) equivale então, a um tipo de queda de pressão, a saber: Aceleração, fricção e gravidade, definidos nas equações (2.1) a (2.3).

$$
\begin{aligned}
& -\left(\frac{d p}{d z}\right)_{a c l}=\frac{d}{d z}\left(\frac{G^{2}}{\rho}\right) . \\
& -\left(\frac{d p}{d z}\right)_{f r i}=\frac{1}{A_{Z}}\left(\int_{P_{Z}} \tau_{w} d P_{Z}\right) .
\end{aligned}
$$

Revista Internacional de Ciências, v. 10, n. 02, p. 3-22, mai-ago, 2020 
$-\left(\frac{d p}{d z}\right)_{\text {grav }}=\rho g$

No presente trabalho, a queda de pressão por aceleração foi desprezada e a queda por friç̧ão foi dividida em duas componentes: Atrito e forma. A queda de pressão por aceleração foi desprezada por ser irrisória nas condições do estudo. Não foi considerado o fluído acelerado por uma bomba e o regime era estacionário. Sendo assim, a queda de pressão no canal ao longo do eixo axial é definida como:

$$
\Delta p=\Delta p^{g r a v}+\Delta p^{f}+\Delta p^{f o r m}
$$

Onde $\Delta p$ é a variação da pressão, $\Delta p^{g r a v}$ a variação da pressão por gravidade, $\Delta p^{f}$ a variação da pressão por atrito e $\Delta p^{\text {form }}$ variação da pressão por forma.

A queda de pressão por gravidade é determinada pela equação (2.3). Já aqueda de pressão por atrito é estabelecida através de uma correlação, segundo Carajilescov (2012) dependente do número de Reynolds (Re):

$$
\Delta p^{f}=\frac{2 \Delta z}{D_{h}} \rho v^{2} f(\mathrm{Re})
$$

Onde $f(\operatorname{Re})=0,341 \mathrm{Re}^{-0,25}$, Re o número de Reinolds, Dh é o diâmetro hidráulico, definido em Fox et al. (2006) e vé a velocidade do fluido refrigerante. Por fim, a queda de pressão por forma é calculada de maneira semelhante à anterior, porém levando-se em conta apenas os pontos onde o fluxo do refrigerante é perturbado, no caso, nas grades espaçadoras das varetas, cuja função é a de sustentá-las, conforme mostrado na figura 3. Para a determinação da queda de pressão nesse caso, considera-se a correlação dependente do fator de forma apresentado por Franco e Carajilescov (2000):

$$
\Delta p_{i}{ }^{\text {form }}=\frac{1}{2} K \rho v^{2}
$$

$$
\text { Onde } K=11,63 \mathrm{Re}^{-0,25} \text {. }
$$



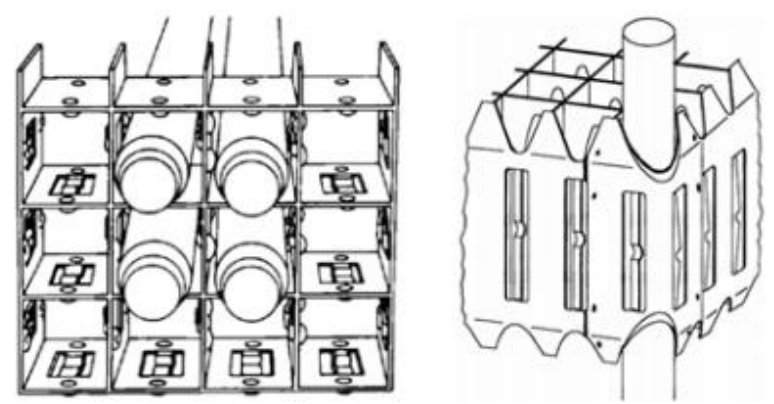

Figura 3. Grades espaçadoras.

Fonte: Todreas e Kazimi (1990).

\section{Análise Térmica}

Trata-se da principal parte do código. Nela, são calculadas as distribuições de temperatura ao longo dos eixos radial e axial do canal mais quente, bem como a distribuição da taxa de DNB (Departure of Nucleate Boilling) e seu valor mínimo. É um fator de suma importância dentro do projeto de desenvolvimento do núcleo de um reator nuclear uma vez que são fatores limitantes dentro do projeto.

0 DNBR, que á a taxa de DNB, em engenharia nuclear, é um fator de garantia de que tal ponto de ebulição não acontecerá com o líquido refrigerante do reator. Sua definição, é dada, conforme Lamarsh e Baratta (2001) por:

$D N B R=\frac{q^{\prime \prime}{ }_{C H F}}{q^{\prime \prime}{ }_{\text {local }}}$

(6)

Sendo q" chғ fluxo de calor crítico, determinado por correlações específicas. Para a concepção do STC-MOX-Th, foi utilizada a correlação W-3, de autoria da Westinghouse, definida no trabalho de Santos (2016). Já q" local é o fluxo de calor ao longo da vareta, analisada em cada ponto dela. A fim de garantir a máxima segurança possível, toma-se como parâmetro o seu menor valor na distribuição (MDNBR). Todreas e Kazimi, estipulam o valor para um ínfimo é de 1,3. Ou seja, que o máximo fluxo de calor ao longo da vareta seja $30 \%$ menor do que o fluxo de calor crítico.

Para a análise térmica do combustível, são consideradas três regiões diferentes, como mostrado na figura 4 : 


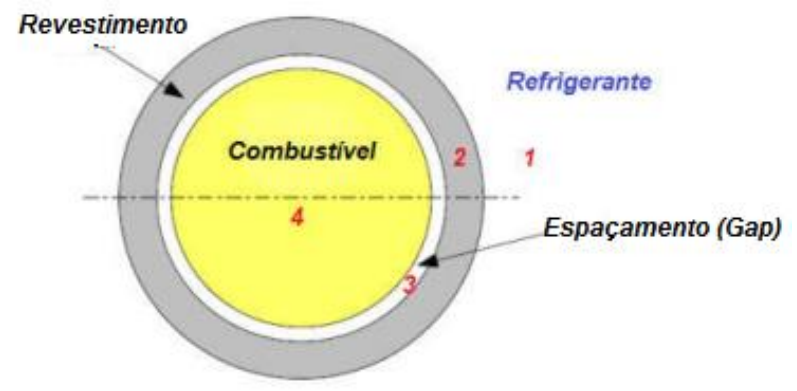

Figura 4. Vista radial da vareta combustível.

Fonte: Santos (2016).

No refrigerante e no espaçamento (gap), que é uma região entre o revestimento e a pastilha combustível preenchida geralmente com Hélio, os cálculos são realizados considerando a conveç̧ão, uma vez que a água leve não é um bom condutor de calor e assim podemos desconsiderar a troca de calor por condução, a principal forma de transferência de calor, utilizando então a lei de resfriamento de Newton, com base em Incropera et al. (2008):

$q^{\prime \prime}=h \Delta T$

Onde (q") é o fluxo de calor produzido, h é o coeficiente de convecção térmica e $\Delta$ T é a variação de temperatura ao longo do eixo radial nos intervalos citados anteriormente. Nas regiões do revestimento e no combustível, o tratamento é feito de maneira a considerar a condução como a forma principal de transferência de calor. Assim, é considerada a equação do calor, em coordenadas cilíndricas, apenas no eixo radial, sob regime estacionário, isto é, independentemente do tempo.

$\frac{1}{r} \frac{d}{d r} k\left(\frac{d T}{d r}\right)+q^{\prime \prime \prime}=0$

Onde (q”") é quantidade de calor produzida por unidade de volume ou quantidade de calor volumétrica e k é a condutividade térmica.

0 tratamento entre as regiões do combustível e do revestimento, apesar de serem baseados na mesma forma de transferência de calor, possuem diferenças. Na região do combustível, a condutividade térmica é uma função da temperatura, isto é, $\mathrm{k}=\mathrm{k}(\mathrm{T})$. Além disso, considera-se a existência de fonte de calor na região (q"”).

Na parte referente ao revestimento, a condutividade térmica é constante. Isso acontece pelo fato da espessura do revestimento ser consideravelmente menor do que o raio da pastilha 
combustível e que a influência da variação da temperatura sobre a condutividade térmica do Zirlo ou Zircaloy, (ligas baseadas em Zircônio utilizadas para o revestimento), é menor do que sobre a condutividade térmica do Urânio e outros actinídeos usados como combustível nuclear, como no trabalho de Santos (2016). Ademais, eventuais fontes de calor nesta região são desconsideradas, o que anula o segundo termo do lado esquerdo da equação (8), o que evidencia ainda mais sua simplificação em relação à região do combustível, que recebeu um tratamento diferenciado no código, cuja descrição será feita adiante. As temperaturas são calculadas nos eixos axiais e radiais, tendo as suas distribuições determinadas conforme referências exemplificadas nas figuras 5 e 6 .

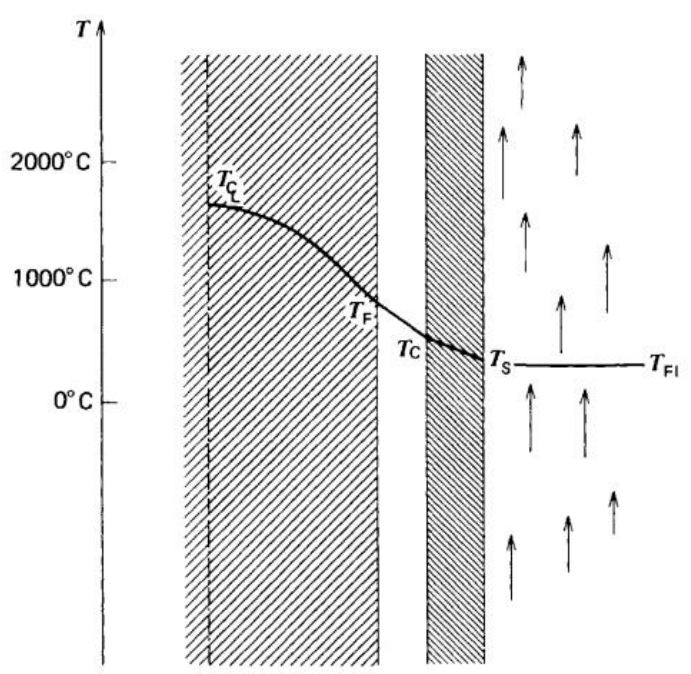

Figura 5. Distribuição de temperaturas ao longo do eixo radial de uma vareta combustível Fonte: Duderstadt \& Hamilton (1976).

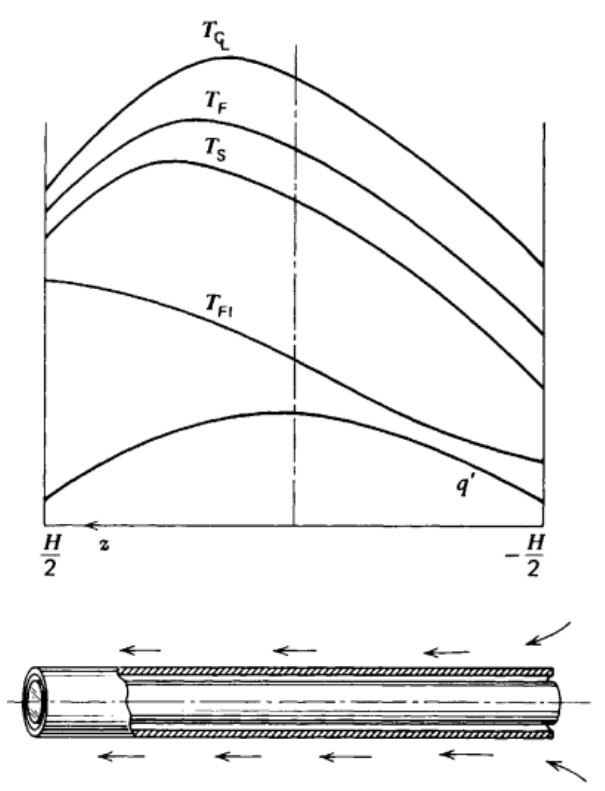

Figura 6. Distribuição de temperaturas ao longo do eixo axial de uma vareta combustível Fonte: Duderstadt e Hamilton (1976). 
Em engenharia nuclear é sabido que a distribuição do fluxo de calor (q”) por toda extensão do eixo axial varia ao longo do período de vida do combustível.

Ou seja, ao longo de sua utilização, que é conhecida como "queima”. 0 período de utilização de uma determinada quantidade de combustível é conhecido como ciclo. Sendo assim, assume-se que, no chamado início de vida (Beginning of cicle - BOC), a distribuição do fluxo seja cossenoidal, como mostrada na equação (9):

$q^{\prime \prime}(z)=q^{\prime \prime}{ }_{M A X} \cos \left(\frac{\pi z}{H_{e}}\right)$

Onde $H_{e}$,definido por Lamarsh e Baratta (2001) é à distância ou comprimento extrapolado.Como o programa é monocanal, qualquer um dos canais presentes no núcleo pode ser analisado. No caso, por uma questão conservativa, o canal escolhido é o dito mais quente, uma vez que se esse canal respeitar os limites de segurança os demais também respeitarão. Para isso é utilizado um fator de pico, chamado de fator de pico nucelar e definido como a razão entre a quantidade de calor volumétrica máxima e a quantidade de calor volumétrica média:

$$
F_{N}=\frac{q^{\prime \prime \prime}{ }_{M A X}}{\overline{q^{\prime \prime \prime}}}
$$

A quantidade de calor por unidade de volume média (q”) é definido como o quociente entre a potência do reator e o volume de combustível. Já em regimes mais avançados de queima, como no Estágio Intermediário de Ciclo (Middle of cicle - MOC) e no Final de Ciclo (End of cicle - EOC) a distribuição do fluxo tende a ficar com uma forma mais achatada. A figura 7 compara a distribuição no início e num estágio mais avançado de queima.

Revista Internacional de Ciências, v. 10, n. 02, p. 3-22, mai-ago, 2020 


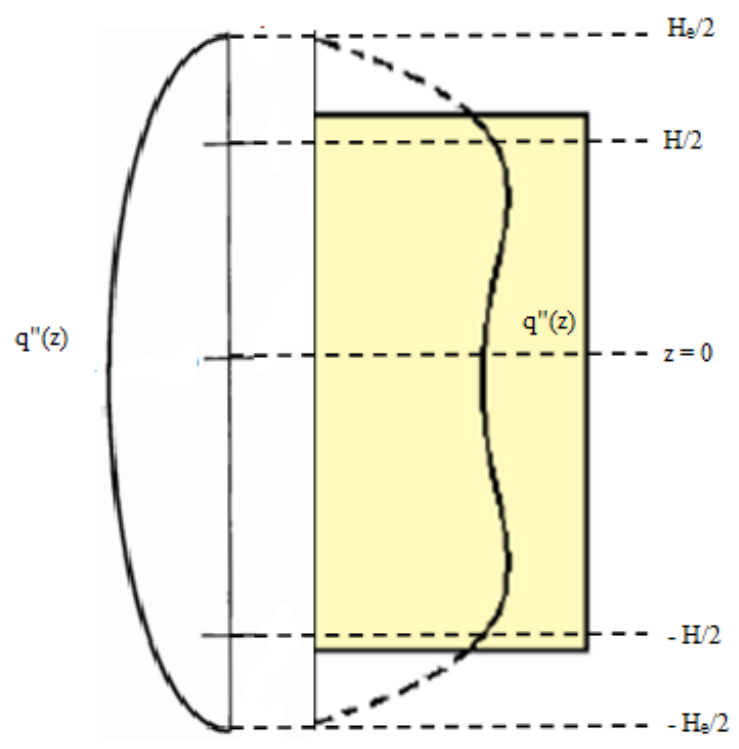

Figura 7. Comparação da distribuição do fluxo de calor q” ao longo da altura z da vareta em diferentes estágios de queima (início de vida na curva a esquerda e ao longo da queima na curva a direita).

Fonte: Santos (2016) - Adaptado

0 achatamento da curva em função do tempo na figura 7 se dá pela maior taxa de reação de fissão em início de vida, devido ao meio fluxo no centro, isso faz com quem o combustível seja mais queimado no centro do que nas extremidades uma vez que a taxa de reação é dada por:

$R=\Sigma_{f} \varphi(\boldsymbol{r})$

Onde R é a taxa de reação dada em reações $/ \mathrm{cm}^{3} . \mathrm{s}, \Sigma_{f}$ é a seção de choque macroscópica de fissão dada por $\mathrm{cm}^{-1}$ e $\varphi(\boldsymbol{r})$ é o fluxo de neutrons dado por neutrons $/ \mathrm{cm}^{2}$. .s.

Dessa forma, o código STC-M0X-Th fornece as distribuições de temperatura, bem como a taxa do DNB, em diferentes estágios de queima, o que é importante tanto dentro da área de projetos de reatores nucleares, quanto na parte de ensino, mostrando as particularidades de cada regime de queima no ponto de vista térmico.

\section{Estrutura Lógica do STC-M0X-Th}

0 STC-MOX-Th tem uma estrutura computacional simplificada, uma vez que seus cálculos se limitam ao uso de um canal. Por questões de segurança, é conveniente que o canal mais quente do núcleo seja o escolhido. Além disso, é considerado que o fluxo do fluido 
refrigerante seja em apenas um eixo. Fluxos em mais de um eixo também podem ser conhecidos por crossflow.

A análise desse canal é feita a partir da distribuição da densidade de potência, que é intimamente relacionada com o fluxo de nêutrons. Grandeza essa fornecida por cálculos neutrônicos, mostrados em Maiorino et al. (2017). A partir desta, juntamente com a vazão mássica do fluido refrigerante e a temperatura de entrada dele, as temperaturas são calculadas radial e axialmente. Em Santos (2016), o canal é subdividido em intervalos axiais e radiais discretos (elementos), de maneira que o cálculo de temperaturas se realize nesses dois eixos simultaneamente. 0 programa permite ao usuário inserir o número que julgar conveniente de elementos, mas a configuração padrão (default) utiliza 98 elementos. A figura 8 mostra o diagrama lógico do programa.

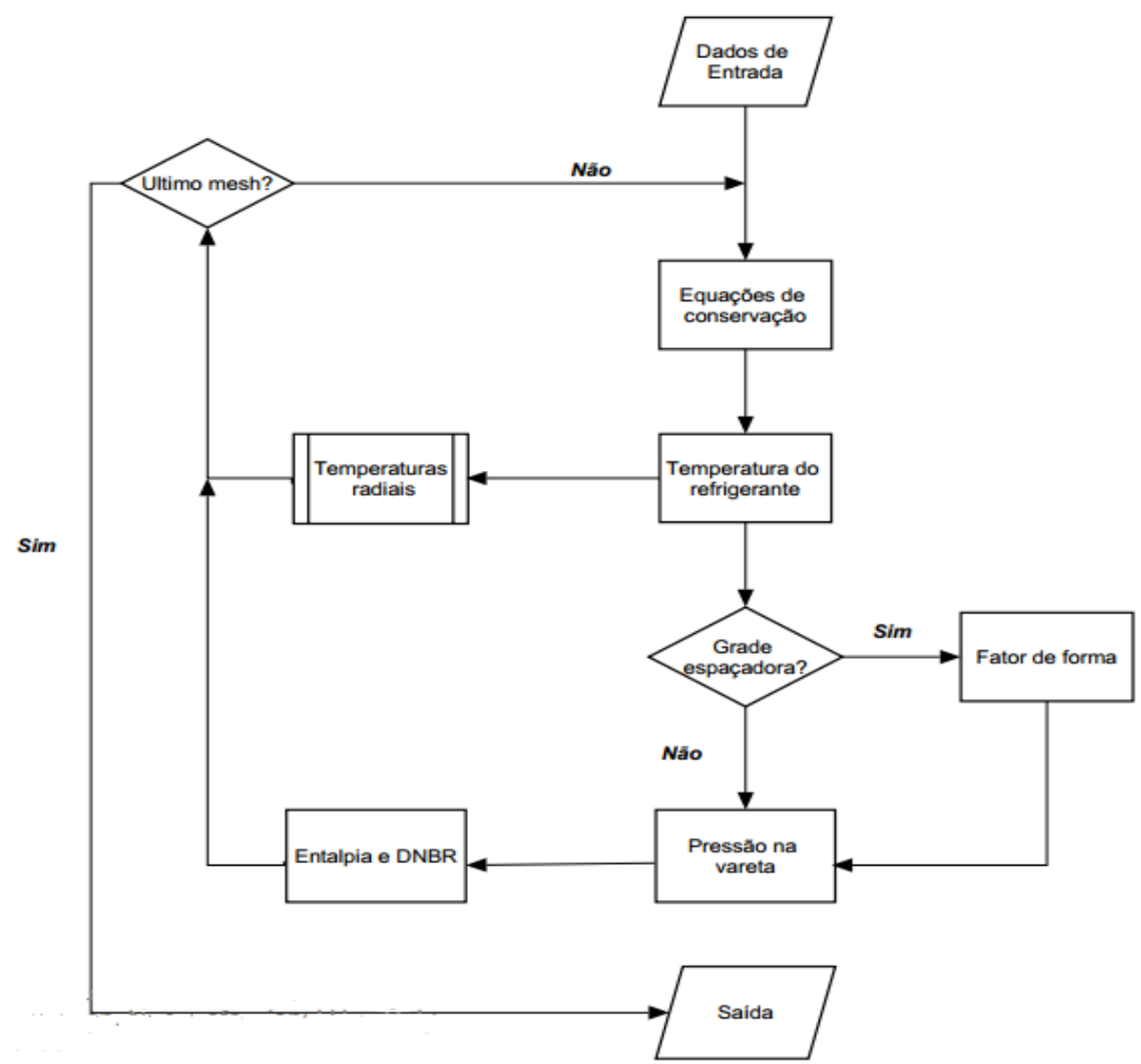

Figura 8. Diagrama lógico do STC-M0X-Th.

Fonte: Santos et al. (2016) - Adaptado.

Em cada mesh os cálculos começam com as equações de conservação de momento e massa, passam pela temperatura do refrigerante naquele ponto. Na sequência, a pressão, considerando ou não a presença das grades espaçadoras e a determinação das entalpias e títulos, através de uma tabela termodinâmica desenvolvida exclusivamente para linguagem 
MATLAB, desenvolvida por Holmgreen (2006). Após esse processo, um novo mesh é iniciado até que toda a vareta tenha sido percorrida. Em especial fica a parte referente ao cálculo da temperatura na pastilha combustível, uma vez que nesta parte, a equação (7) é calculada considerando a condutividade térmica como uma função da temperatura, isto é, $k=k(T)$. Para isso, foi utilizado o método dos elementos finitos, que pode ser visto com mais detalhes no livro de Reddy e Gartling (2010). A discretização é feita considerando a pastilha unidimensional, porém em coordenadas cilíndricas.

Vale ressaltar que, em relação à correlação utilizada para a condutividade térmica do combustível $(k(T))$, há um fator complicador adicional quando a análise se volta para o caso dos combustíveis MOX. Uma vez que as correlações existentes estão fixas em proporções prédeterminadas de porcentagem de massa $\mathrm{U}_{2}$ para $\mathrm{Th}_{2}$. Um estudo é feito sobre a possibilidade da utilização da média ponderada entre as correlações de $k(T)$ tanto do $\mathrm{UO}_{2}$, quanto do $\mathrm{ThO}_{2} .0$ resultado foi satisfatório e tal metodologia foi empregada no código, as comparações de resultado de proporções de massa de $\mathrm{Th}_{2}$ para $\mathrm{U}_{2}$ no óxido misto foram testados para algumas proporções na dissertação de Santos (2016).

\section{RESULTADOS E DISCUSSÃ0}

Com o objetivo de validar o código STC-MOX-Th, foram utilizados os dados do reator AP1000 , mostrados na tabela 1.

Tabela 1. Dados de entrada utilizados na validação do código STC-MOX-Th

\begin{tabular}{cc}
\hline Grandeza & Valores/Unidade \\
\cline { 2 - 3 } Combustível (material) & $\mathrm{U} 0_{2}$ \\
Revestimento (material) & Zirlo \\
Raio pastilha combustível & $4,090 \mathrm{~mm}$ \\
Espessura gap & $0,082 \mathrm{~mm}$ \\
Espessura revestimento & $0,57 \mathrm{~mm}$ \\
Temperatura entrada & \\
(refrigerante) & $279,4{ }^{\circ} \mathrm{C}$ \\
Vazão mássica & $14300 \mathrm{~kg} / \mathrm{s}$ \\
Fluxo mássico & $3458 \mathrm{~kg} / \mathrm{m}^{2} \mathrm{~s}$ \\
Pitch & $12,6 \mathrm{~mm}$ \\
$\mathrm{~N}^{0}$ de varetas & 289 \\
Máxima densidade linear de & $512 \mathrm{~W} / \mathrm{cm}$ \\
potência & $4260 \mathrm{~mm}$ \\
Comprimento vareta & $155 \mathrm{bar}$ \\
Pressão de entrada & 157 \\
$\mathrm{~N}^{0}$ de assemblies &
\end{tabular}

Fonte: Westinghouse (2011). 
Neste trabalho foram feitas medidas tanto da parte térmica, quanto da parte hidráulica, com o intuito de permitir ao leitor a maior compreensão possível das capacidades do programa, tanto no que diz respeito ao ensino, quanto à pesquisa. A metodologia de análise é mostrada na figura 9 .
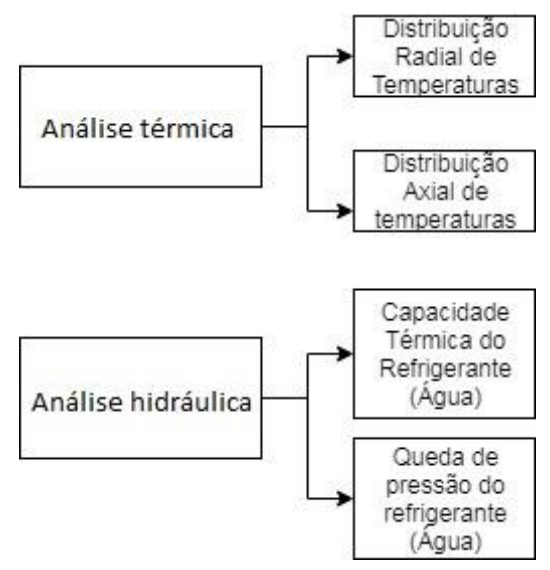

Figura 9. Esquema de análise do trabalho Fonte: Santos et al. (2016) - Adaptado.

\section{Análise Hidráulica}

Na primeira parte do trabalho, focou-se apenas na análise das grandezas referentes ao fluido refrigerante do reator, neste caso, a água. As grandezas estudadas eram funções dependentes do comprimento da vareta. Nesta seção, a densidade, a pressão, a capacidade térmica e a entalpia foram calculadas.

Gráfico 1. Densidade e pressão da água como função do comprimento da vareta combustível.

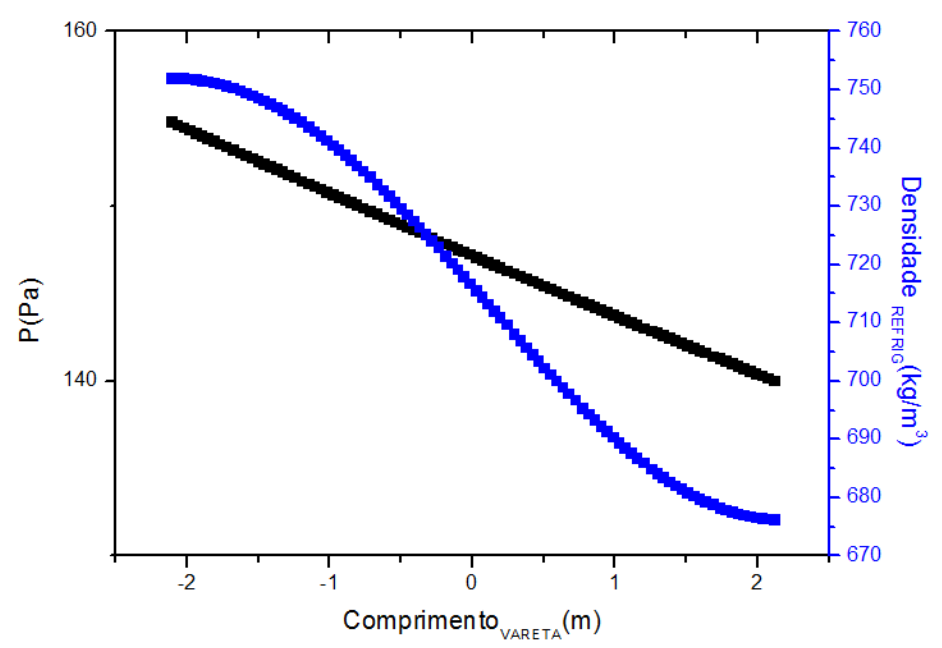

Fonte: Autor (2019). 
0 gráfico mostra bem o comportamento físico das duas grandezas em questão já previamente esperado: A queda de pressão de maneira linear e a densidade decaindo exponencialmente. Não ficam claros os pontos onde há queda de pressão por conta das grades espaçadoras.

Gráfico 2. Capacidade térmica e Entalpia da água como função do comprimento da vareta combustível.

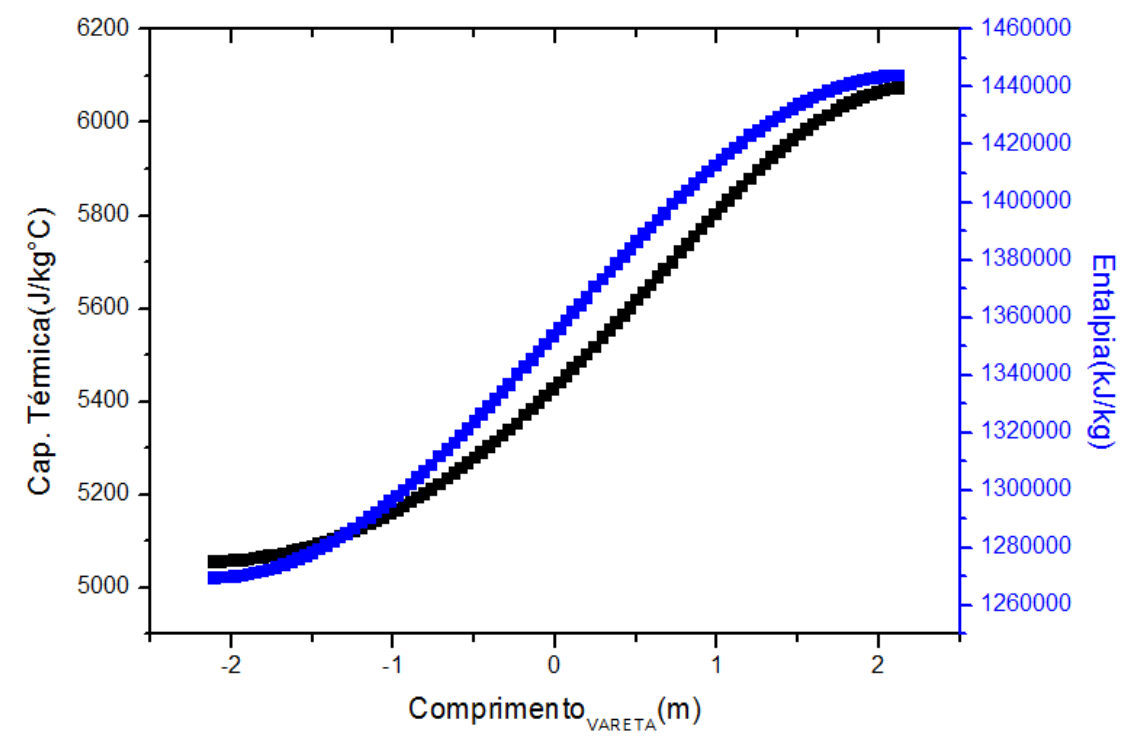

Fonte: Autor (2019).

\section{Análise Térmica}

Já na segunda parte da análise, o enfoque foi voltado aos cálculos da temperatura da pastilha combustível, que é um importante fator de projeto e segurança de um reator nuclear PWR. Os gráficos 3 e 4 tratam da questão da temperatura. No gráfico 3, é feita a análise da temperatura do centro da pastilha para diferentes proporções do MOX de (U,Th). Já no gráfico 4, a análise é voltada a diferentes estágios de queima do combustível numa mesma proporção do MOX. Sendo estes estágios início de vida (BOC), que equivale ao momento que o reator começou a operar com combustível novo, meio de vida (M0C), que equivale a 225 dias de operação e final de vida (EOC) que equivale a 450 dias de operação. 
Gráfico 3. Temperaturas no centro da pastilha combustível para diferentes proporções do MOX (U,Th)

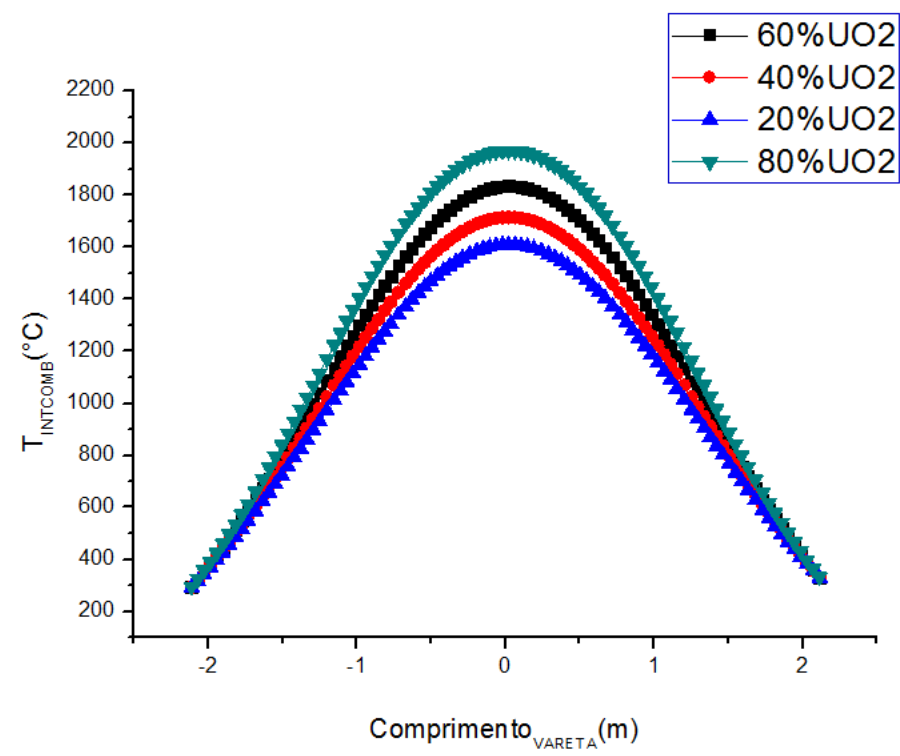

Fonte: Autor (2019).

Gráfico 4. Temperaturas no centro da pastilha combustível em diferentes estágios de queima.

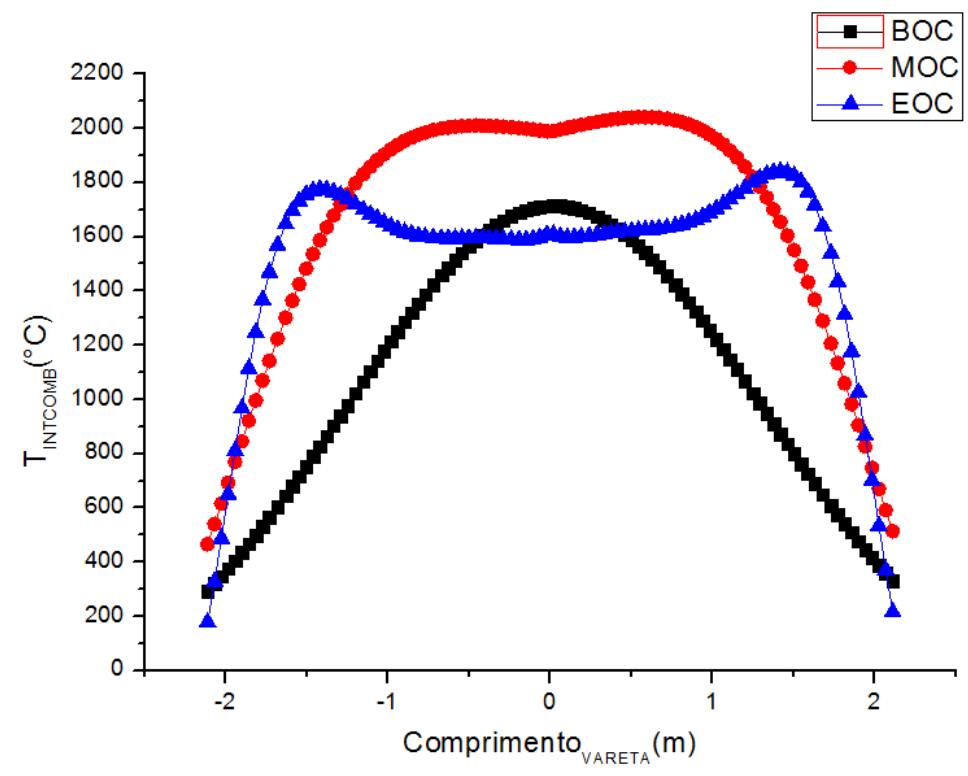

Fonte: Autor (2019).

0s resultados se mostraram condizentes quando validados com os relatórios fornecidos pela Westinghouse (2011). As grandezas hidrodinâmicas também se comportaram dentro do esperado, como a queda de pressão linear ao longo da vareta. Uma comparação dos resultados do código também foi realizada para modelagem de óxidos mistos de tório e urânio utilizando o código comercial CFX e o trabalho de Cunha et al. (2019) demonstrando resultados compatíveis. Tais resultados demonstraram que apesar de compatíveis, o STC-MOX-Th superestima levemente as temperaturas $(\sim+3 \%)$ no combustível as temperaturas obtidas nestes 2 trabalhos 
estão dispostas na tabela 2. Esse fato não prejudica análises feitas com o código, uma vez que o principal objetivo das análises térmicas do combustível é evitar que a temperatura se aproxime do ponto de fusão. Assim uma análise com temperaturas superestimadas dentro dos limites de segurança contribuirá ainda mais para distanciar destes.

Tabela 2. Comparação de dados do código STC-MOX-Th e de dados do CFX

\begin{tabular}{ccc}
\hline Grandeza & CFX & STC-M0X-Th \\
\hline $\begin{array}{c}\text { Temperatura de saída da água no } \\
\text { canal quente APTh1000 } \\
\begin{array}{c}\text { Temperatura no centro da } \\
\text { pastilha de combustível no canal } \\
\text { quente APTh1000 }\end{array}\end{array}$ & $317{ }^{\circ} \mathrm{C}$ & $327{ }^{\circ} \mathrm{C}$ \\
& $1576{ }^{\circ} \mathrm{C}$ & $1615^{\circ} \mathrm{C}$
\end{tabular}

Fonte: Cunha (2019).

Note que se a temperatura de operação segura é garantida para o STC-MOX-Th, como ele superestima as temperaturas, o reator ficará inerentemente mais seguro.

É importante notar que o AP-1000 tem o $\mathrm{UO}_{2}$ puro como combustível. A utilização do $\mathrm{Th}_{2}$, graças as suas características termofísicas, garante um ponto de fusão mais alto e temperaturas centrais do combustível mais baixas. Trabalhos mostram que o STC-MOX-Th tem resultados conservativos, porém preservando as suas características físicas.

0 STC-MOX-Th tem sido aplicado em alguns trabalhos científicos como o publicado por Maiorino, et al. (2017), Stefani et al. (2019) e Stefani et al. (2020).

\section{CONCLUSÃO}

A confiabilidade do código foi verificada através de trabalhos publicados em congressos e na dissertação do autor principal, conforme mencionado no texto. Foram realizadas comparações com o código CFX, que demonstraram uma diferença da ordem de 3\% no valor das temperaturas obtidas no centro da pastilha de combustível mais quente. 0 código tem sido aplicado em alguns estudos desenvolvidos pelo grupo de estudos de tório da Universidade Federal do ABC, com publicações em revistas e alto fator de impacto conforme mencionado no texto.

0 STC-M0X-Th, mostrou-se extremamente versátil, uma vez que o fato de ser conservativo garante a segurança de projetos de reatores PWR e ao preservar de maneira geral a fenomenologia física do problema, bem como a boa interface com o usuário, o torna uma 
ferramenta interessante no processo de aprendizagem na área de termo hidráulica de reatores nucleares. Soma-se a isso ainda o fator inovador de permitir a utilização de diferentes proporções do combustível MOX (U,Th), o que acarreta ganhos tanto na pesquisa, quanto no ensino.

Para um aprimoramento futuro, a inserção de módulos que calculem outros tipos de combustíveis, como o MOX (U,Pu) - Urânio/Plutônio - seria um ponto interessante.

\section{REFERÊNCIAS BIBLIOGRÁFICAS}

Belhot, R.; Figueiredo, R.;Malavé, C. 0 Uso da Simulação no Ensino de Engenharia. XXIX Congresso Brasileiro de Ensino de Engenharia. Porto Alegre, 2001.

Carajilescov, P. Introdução a Engenharia Nuclear - Notas de aula. Santo André: UFABC, 2012.

Cunha, C. J.; Rodriguez, D. G., Lira, C. A.; Stefani, G. L.; Lima, F. R. THERM0HYDRAULIC ANALYSIS OF A FUEL ELEMENT OF THE AP1000 REACT0R WITH THE USE OF MIXED OXIDES OF U / Th USING THE COMPUTATIONAL FLUID DYNAMIC CODE (CFX). INAC 2019 - Nuclear New Horizons: Fueling our Future. Santos, 2019.

Duderstadt, J. J.; Hamilton, L. J. Nuclear Reactor Analysis. John Wiley \& Sons, 1976.

Fox, R. Introdução a mecânica dos fluidos. LTC, 2006.

Franco, C.; Carajilescov, P. Experimental analysis of pressure drop and flow redistribution in axial flows in rod bundles. Journal Brazilian Society of Mechanical Sciences and Engineering, vol.22, $\mathrm{n}^{\circ} 4,2000$.

Holmgreen, M. X-Steam for MATLAB - User's Manual, 2006.

Incropera, F., Dewitt, D. P., Bergman, T., \& Lavine, A. Fundamentos de Transferência de Calor e Massa. Rio de Janeiro: LTC, 2008.

Junior, J. Cálculo da distribuição de temperatura em varetas combustíveis: Estudo do efeito da excentricidade no posicionamento das pastilhas de U02. Rio de Janeiro, RJ: Dissertação de Mestrado/IEN, 2000.

Lamarsh, J. R., EBaratta, A. Introduction to Nuclear Engineering. Prentice Hall (2001).

Maiorino, J. R.;Stefani, G. L.; Moreira; J. M. L., Rossi, P. C. R.; Santos, T. Feasibility to convert an advanced PWR from U02 to a mixed U/Th02 core - Part I: Parametric Studies. Annals of Nuclear Energy, 102, pp. 47-55, 2017

Masterson,R. E., Nuclear Engineering Fundamentals - A practical perspective. EUA: Taylor \& Francis Group, LLC, 2017.

Reddy, J., \& Gartling, D. The finite element method in heat transfer and flud dynamics (3rd edition). CRC Press, 2010.

Rowe, D. COBRA II: A Digital Computer Program for Thermal Hydraulic Subchannel Analysis of Rod Bundle Nuclear Fuel Elements. BNWL-1229, 1970.

Santos, T. A. Desenvolvimento de um código mono canal para análise termo hidráulica de reatores PWR. Santo André, SP: Dissertação de Mestrado/ UFABC, 2016.

Revista Internacional de Ciências, v. 10, n. 02, p. 3-22, mai-ago, 2020 
Santos, T.A., Maiorino, J. R., \& Laranjo, G. On the thermal limits for PWR calculation in operational conditions using a mono channel, mono phasic model for mixed thorium-uranium oxide fuel. 16th Brazilian Congress of Thermal Sciences and Engineering - ENCIT 2016. Vitória, ES, Brasil: ABCM, 2016.

Santos, T.A., Maiorino, J. R., \& Laranjo, G.. A thermal hydraulic analisys in PWR reactors with U02 or (U-Th)02 fuel rods employing a simplified code. International Nuclear Atlantic Conference - INAC 2017. Belo Horizonte, MG, Brasil: 2017.

Stefani, G. L.; Moreira, J. M. L.; Maiorino, J. R. \& Rossi, P. C. R.. Detailed neutronic calculations of the AP1000 reactor core with the Serpent code. Progress in Nuclear Energy, vol 116, pp. 95-107, 2019.

Stefani, G. L.; Maiorino, J. R. \& Moreira, J. M. L.. The AP-Th 1000 - An advanced concept to use MOX of thorium in a closed fuel cycle. International Journal of Energy Research, vol 2020, pp. 1-14, 2020.

Todreas, N., GKazimi, M. Nuclear Systems I - Thermal Hydraulic Fundamentals. EUA: Taylor \& Francis, 1990.

USNRC.United States Nuclear Regulatory Commission. Typical Pressurized Water Reactor 2017. Disponível em https://www.nrc.gov/reactors/operating/ops-experience/vessel-head-degradation/vessel-head-degradationfiles/pwr-rx-vessel.html. Acessado em 8 de dezembro de 2019.

Westinghouse. Westinghouse AP1000 Design Control Document - Rev.19, Tier 2, Charapter 4 - Reactor - Section 4.4 Thermal and Hydraulic Design, 2011.

Zernick, W., Currin, H. B., Elyath, E., EPreviti, G. THINC-A Thermal Hydraulic Interaction Code for Semi-0pen or Closed Channel Cores. WCAP-2704,Westinghouse Electric Corporation, 1962. 